\title{
Recent developments in automatic scoring of rodent sleep
}

\author{
S. BASTIANINI' , C. BERTEOTTI' ', A. GABRIELLI², V. LO MARTIRE' ${ }^{1}$ A. SILVANI' \\ G. ZOCCOLI'.
}

'PRISM lab, Department of Biomedical and Neuromotor Sciences; ${ }^{2}$ Department of Physics and Astronomy, Alma Mater Studiorum, University of Bologna, Bologna, Italy

\begin{abstract}
A B S T R A C T
Sleep research carried out on rat and mouse model led to the publication of more than 5000 papers in the last 15 years*, of which more than 500 in 2014. Wake-sleep scoring represents a crucial step of the work performed in preclinical sleep laboratories; it is a time consuming task and a potential source of errors affecting research outcomes. Several algorithms have been developed to perform automatic sleep scoring. Automatic scoring can accelerate the work of researchers substantially. Moreover, the use of sleep scoring algorithms facilitates the direct comparison of the results produced in different laboratories, with clear advantages from the viewpoint of the advancement of science and reduction of the number of animals used for research.

The intent of this review is to provide the readers with the last developments in scoring in rodent sleep and to stress about the need of a cross-lab and cross-species validated algorithm.
\end{abstract}

"Pubmed (http://www.ncbi.nlm.nih.gov/pubmed) records indicate 5322 papers including the words "sleep" and either "rat"/"rats" or "mouse"/"mice" in the title or abstract published between 2000 and 2014, with 513 papers published in 2014 (query made on July 25th 2015).

\author{
Key words \\ Algorithm $\bullet$ Scoring $\bullet$ Sleep $\bullet$ Mice $\bullet$ Rats
}

\section{Sleep as a topic of growing interest in biomedical research}

The study of the physiology and pathophysiology of the wake-sleep cycle is an expanding research field. The elevated number of sleep disorders undoubtedly fuels this interest. According to the International Classification of Sleep Disorders of the American Academy of Sleep Medicine (Sateia, 2014), 95 different sleep disorders have been identified until now. These disorders were grouped into 7 main classes: insomnia, sleep-related breathing disorders, central disorders of hypersomnolence, circadian rhythm sleep-wake disorders, parasomnias, sleep- related movement disorders and other sleep disorders. Among these groups are included conditions such as narcolepsy, hypersomnia, sleep apneas, snoring, bruxism, periodic limb movements, REM sleep behavior disorders, shift-work, sleepwalking and sleep paralysis. From the scientific viewpoint, the study of the wake-sleep cycle and its alterations is of interest to molecular biologists (genetic and epigenetic alterations; cf. Dauvilliers and Tafti (2008); Palagini et al. (2015); Paterson et al. (2011); Toth and Bhargava (2013) for recent examples) in addition to physiologists and neuroscientists (autonomic, metabolic, cardiovascular, respiratory, thermoregulatory, motor, inflammatory systems; cf. Amici et al. (2014); Dengler et al. (2015); Floras 
(2015); Romeijn et al. (2012); Spiegel et al. (2009) for recent examples). In summary, sleep research is at the intersection among different areas of medicine and basic science, explaining why this field is constantly expanding.

\section{Mice and rats in sleep research}

The use of animal model is critical in biomedical research because the level of complexity of the interactions between molecules, cells, organs and systems can hardly be understood by looking at the single components. Moreover, animal models allow researchers to test the safety and efficacy of new therapies before applying them on human subjects (Paterson et al., 2011). Among animal models, rodents offer several advantages: rats and mice are relatively cheap to buy and maintain, their size is small and their life cycle is short (Paterson et al., 2011; Toth and Bhargava, 2013). Research on mice has received impulse after 2002, when mouse DNA was completely sequenced (Mouse Genome Sequencing et al., 2002). This has allowed the development of hundreds of genetically-modified mouse strains that have been used in many different research areas. In particular, the development of genetically-modified mouse models reproducing specific sleep disorders accelerated the understanding of these diseases and their correlates (Toth and Bhargava, 2013). A successful example was the generation of two engineered mouse models, in which two different genetic approaches were applied to stop the production of the orexin peptides or to selectively kill the orexinergic neurons (Chemelli et al., 1999; Hara et al., 2001). These geneticallymodified mice show a narcoleptic phenotype very closed to those of human patients, and have been key to foster the understanding of narcolepsy pathophysiology. For example, cardiovascular derangements during sleep (Bastianini et al., 2011) and electroencephalographic anomalies during cataplexy (Bastianini et al., 2012) have been discovered on these mice and later translated to human patients with narcolepsy/cataplexy (Grimaldi et al., 2012; Vassalli et al., 2013).

In addition to mice, rats are useful models especially in sleep research because their bigger size (rats are 10 times heavier than mice) allows scientists to more easily study specific brain areas by means of electrophysiological and pharmacological manipulations (cf. Cerri et al. (2013); Luppi et al. (2013) for recent examples). Indeed, most of the experiments showing the effects on sleep of specific neurotransmitters, such as histamine, adenosine, and serotonin, and their receptors have been performed on rats (cf. Bergmann et al. (1987); Sharma et al. (2014) for relevant examples). Pharmacological and immunohistochemical studies on rat brains significantly contributed to our understanding of the brain circuits involved in the regulation of the wake-sleep cycle (Luppi et al., 2013).

The translation of results obtained on rats and mice to human subjects has some limitations. First, rodents are nocturnal animals with polyphasic distribution of sleep (NREM and REM) stages. Wakesleep cycle is thus more fragmented in rodents than in human subjects and the sleep phase in rodents is more largely represented by NREM sleep than in human subjects. Finally, in human subjects, the NREM sleep state is typically divided in 3 to 4 different stages (Paterson et al., 2011; Toth and Bhargava, 2013). Conversely, although a distinct pre-REM sleep state has been described in mice (Glin et al., 1991) and rats (Gottesmann, 1996), NREM sleep is generally not divided in stages in rodents. Despite these discrepancies, rodents and human subjects share many electroencephalographic (EEG) and regulatory characteristic of wake-sleep cycle. A more substantial yet rarely appreciated limitation is that despite the intensive use of mice and rats in basic sleep research, a large consensus on the criteria to discriminate wake-sleep states in rodents is still missing. Explicit and quantitative consensus criteria would represent a great advantage to compare and interpret results across different laboratories and species. The lack of a common method of sleep analysis undoubtedly contributes to discrepancies among results published by different research teams. This issue has long been recognized as critical for human sleep research. Accordingly, the American Academy of Sleep Medicine has recently designed a web page, where users can score sleep states based on raw tracings and compare the results with those of a golden standard (http://www. aasmnet.org/isr/). It would be extremely useful to extend this procedure also to data obtained on animal models. 


\section{Manual Vs. Automatic Sleep Scoring}

Usually, sleep studies on either human subjects or rodents are performed on the basis of the EEG and electromyogram (EMG) activity. Due to the technical complexity associated with the small size of rodents, the electrooculogram (EOG) is recorded on human subjects but usually not in these species (Ronzhina et al., 2012). As the time spent to score sleep is proportional to the duration of the recording period, a growing interest in reducing human intervention in the scoring procedure is spreading among the sleep research community. Several commercial systems for automatic sleep scoring in humans are already available. Unfortunately, all these systems contain decision algorithms which are based on the Rechtschaffen and Kales scoring rules thus splitting NREM sleep into 4 distinct stages. Moreover, some of them need the EOG signal to correctly perform the sleep discrimination (Ronzhina et al., 2012). For these reasons, automatic algorithms for sleep scoring in humans can not be directly used in basic sleep research.

Consequently, numerous automated systems for sleep scoring in rodents have been developed and validated (Brankack et al., 2010; Robert et al., 1999; Rytkonen et al., 2011; Sunagawa et al., 2013; Veasey et al., 2000). Nonetheless, most basic animal sleep laboratories still construct hypnograms based on visual inspection of polygraphic recordings performed by expert investigators (El Helou et al., 2013; Gondard et al., 2013; Kantor et al., 2013). Manual scoring of sleep states is thought to ensure precise characterization of wake-sleep behavior, and has the undeniable advantage to promote the identification of unexpected EEG events (Bastianini et al., 2011; Silvani et al., 2014). On the other hand, manual sleep scoring is lengthy, causing a marked slowdown in productivity, and the unavoidable subjectivity of wake-sleep state discrimination may hinder the replication of results. Finally, training investigators to discriminate wake-sleep states is a long process, which can limit but hardly avoid variability between different researchers, even in the same team (Ronzhina et al., 2012).

The development of automatic sleep scoring algorithms based on consensus criteria, robustly validated across different laboratories and possibly applicable to different species would be of great help for the sleep community. These algorithms would acceler- ate sleep studies, improve replicability of results by avoiding subjectivity in sleep discrimination, and possibly be applied in real time for sleep deprivation protocols (Libourel et al., 2015; Vivaldi and Bassi, 2006). Sleep scoring algorithms may also combine conventional discrimination of wake-sleep states with novel techniques of sleep EEG analyses, such as the cluster state-transition analysis (Diniz Behn et al., 2010), fostering new discoveries in sleep research.

\section{RecentValidated Algorithms for Automatic Sleep Scoring}

The firsts algorithms for automatic sleep scoring in rodents were proposed in the ' 70 (Kohn et al., 1974). Since then, new and increasingly evolved algorithms, either commercial or open-source, have been proposed (Brankack et al., 2010; Rytkonen et al., 2011; Sunagawa et al., 2013; Veasey et al., 2000). Automatic sleep scoring algorithms can be divided into two main groups: unsupervised and supervised. Unsupervised algorithms discriminate sleep states using rules such as Bayesan probability or artificial neural network classification methods defined before the staging process. Supervised algorithms need human interactions to manually set wake-sleep state cutoffs or to manually score a subset of data for each subject before proceeding to the analysis of the remaining data. Therefore, unsupervised algorithms typically result less flexible to different recording setups and they hardly perform satisfactorily on outliers subjects, whereas supervised algorithms inevitably suffer to some extent from subjectivity. Most of the more recent automatic algorithms belong to the latter group (Brankack et al., 2010; Crisler et al., 2008; Gross et al., 2009; Kreuzer et al. 2015; Louis et al., 2004; Rytkonen et al., 2011; Veasey et al., 2000). Fewer unsupervised algorithms have been recently proposed but they resulted computationally intensive (Sunagawa et al., 2013) and have been tested on a limited number of animals (Libourel et al., 2015; Sunagawa et al., 2013; Stephenson et al., 2009; Costa-Miserachs et al., 2003). Finally none of these algorithms (Table I), even those showing very good performance (Crisler et al., 2008; Kreuzer et al. 2015; Rytkonen et al., 2011; Sunagawa et al., 2013), have been tested on independent datasets (i.e. crosslaboratory validation) (Rytkonen et al., 2011) and different species (Brankack et al., 2010; Crisler et al., 
2008; Gross et al., 2009; Libourel et al., 2015; Louis et al., 2004; Sunagawa et al., 2013; Stephenson et al., 2009; Costa-Miserachs et al., 2003).

\section{An example of cross-laboratory and cross- species validated automatic algorithm for sleep scoring: SCOPRISM}

Recently, we proposed a new open-source algorithm (Matlab) for sleep scoring in mice called SCOPRISM (Bastianini et al., 2014). Briefly, SCOPRISM operates in two main steps with a changeable time resolution, with a default epoch length of $4 \mathrm{~s}$. Initially, sleep scoring is performed according to two local properties of each recorded epoch: the ratio between EEG spectral power in the $\theta(6-9 \mathrm{~Hz})$ and $\delta(0.5-4 \mathrm{~Hz})$ frequency ranges, and the root mean square (rms) of the EMG signal. In the second step, a series of rules that take into account not only the local EEG/EMG properties but also the information on EEG and EMG in adjacent epochs, are used to refine the initial scoring. The fraction of epochs characterized by each given combination of the two local properties (EEG $\theta / \delta$ ratio and EMG rms) is plotted in the first step as 3D surface (Figure 1). This plot typically included a peak at high values of EMG rms, which corresponds to episodes of wakefulness, and a second peak at low EMG rms and EEG $\theta / \delta$ ratio values corresponding to episodes of NREM sleep. Finally, REM sleep corresponds to epochs with low EMG rms and high EEG $\theta / \delta$ ratio values. SCOPRISM automatically finds the EMG rms values to discriminate between wakefulness and sleep states and adapts it to each new subject. On the other hand, the discrimination between sleep states (NREM and REM sleep) is performed using a fixed value of the EEG $\theta / \delta$ ratio equal to 1 , meaning equal EEG spectral power in the theta and delta frequency ranges, which are prominent during REM sleep and NREM sleep in rodents, respectively.

SCOPRISM validation was performed on a large population of mice (including different strains of genetically-modified mice) that allowed us to detect some limits of this algorithm (Bastianini et al., 2014). However, we took advantage of the identification of the rare outliers and developed a flow-chart with specific rules for the correct application of SCOPRISM. Moreover, for the first time we performed a crosslaboratory (analyzing and comparing data of mice recorded in 3 different laboratories) and cross-species (analyzing and comparing data recorded on mice and rats) validation of our algorithm (Bastianini et al., 2014). The overall accuracy, specificity and sensitivity values of the internal validation of SCOPRISM $(97 \%, 95 \%$, and $94 \%$, respectively) were very similar with those calculated on mouse data from different laboratories ( $1^{\text {st }}$ lab: $96 \%, 97 \%$ and $97 \%$, respectively; $2^{\text {nd }}$ lab: $92 \%, 91 \%$ and $88 \%$, respectively) and on rats $(89 \%, 87 \%$, and $95 \%$, respectively).

One of the most interesting peculiarities of this algorithm is that even though it is a fully automatic (unsupervised) system, it allows experimenters to perform a post-hoc supervision. Indeed, together with a matrix containing the automatic sleep scoring, SCOPRISM also provides the 3-D plot of the epoch distribution and a second plot indicating the specific EMG boundary region used for the mouse under study. On the base of these 2 plots, following the flow-chart we proposed, the experimenters can evaluate SCOPRISM reliability before looking at the results of the sleep analysis. This characteristic is novel for an automatic sleep-scoring algorithm and may be particularly useful because it ensures objectiveness, adaptability to each experimental subject and prevention of possible bias introduced by human intervention. Finally, SCOPRISM is the automatic sleep scoring algorithm with the highest overall (considering wakefulness, NREM and REM sleep states together) accuracy (agreement) when compare to manual scorer (Table I).

In light of these characteristics, we believe that SCOPRISM and algorithms with similar properties (open-source, post-hoc supervised, and validated across laboratories and species) may significantly help automate and standardize sleep scoring in rodents. It must be stressed that no algorithm is perfect and definitive in itself, and a large consensus is mandatory before accepting an algorithm as a standard research tool. In line with this view, it is critical that algorithms such as SCOPRISM are made freely available so that researchers in other laboratories apply them to their own raw data and improve them in this endeavor.

\section{Final Summary}

Sleep is a growing area of interest because this physiological behavior is a common topic in many different areas of biomedical research (Sateia, 2014). 


\section{A}
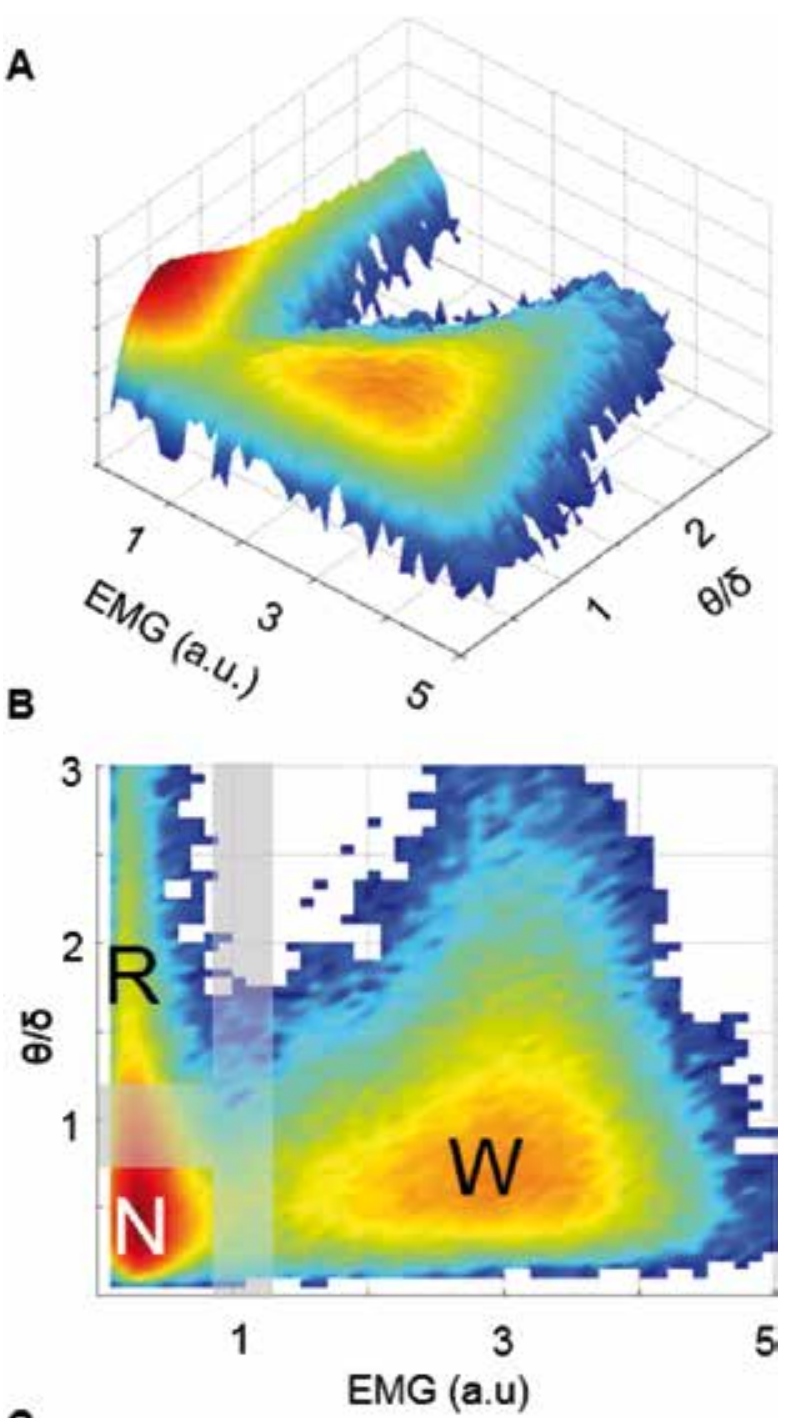

C

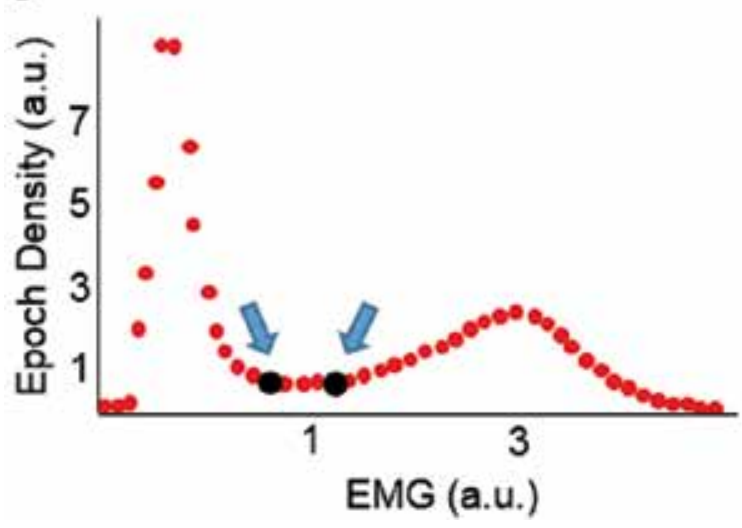

Fig. 1. - Panel A shows the typical distribution profile of recorded 4-s epochs as a function of their electromyogram (EMG) root mean square values (x axis) and on the ratio between $\theta(6-9 \mathrm{~Hz})$ and $\delta(0.5-4 \mathrm{~Hz})$ spectral power of the electroencephalogram (EEG, y axis). On the $z$ axis, a colorimetric scale represents the fraction of epochs characterized by each given combination of EMG and EEG values. For the sake of clarity, the y axis scale was truncated at $\theta / \delta=3$. Panel B shows a bidimensional view of the same epoch distribution. Gray areas correspond to boundary regions (undetermined state attribution), which are used by the algorithm to discriminate between wakefulness (W), non-rapid-eye-movement sleep (N) and rapid-eye-movement sleep (R). Panel C shows a bidimensional plot of the fraction (density) of 4-s epochs as a function of EMG only; arrows indicate the limits of the boundary region between W and sleep states. Reproduced from (Bastianini et al., 2014), with permission. 


\begin{tabular}{|c|c|c|c|c|c|c|c|c|c|c|c|c|}
\hline 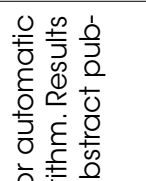 & 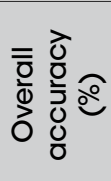 & $\begin{array}{l}m \\
\stackrel{2}{\alpha}\end{array}$ & $\stackrel{m}{\dot{\alpha}}$ & $\begin{array}{l}\infty \\
\infty\end{array}$ & 2 & $\triangleright$ & $\hat{\not \alpha}$ & $\approx$ & $\begin{array}{l}\circ \\
\dot{\alpha}\end{array}$ & $\stackrel{\circ}{\mathscr{\sigma}}$ & $\stackrel{\nabla}{\stackrel{\sigma}{\sigma}}$ & $\stackrel{20}{\alpha}$ \\
\hline 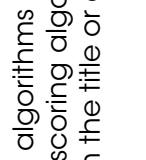 & $\begin{array}{l}\frac{}{\circ} \\
\frac{0}{0} \\
\frac{0}{0} \\
\frac{0}{\overline{0}}\end{array}$ & $\begin{array}{l}\frac{0}{0} \\
\frac{1}{0} \\
\frac{\underline{\underline{T}}}{\underline{\underline{T}}}\end{array}$ & 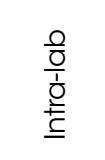 & $\begin{array}{l}\frac{0}{\frac{0}{O}} \\
\frac{1}{0} \\
\underline{\underline{\underline{I}}}\end{array}$ & 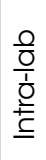 & $\begin{array}{l}\frac{0}{0} \\
\frac{0}{0} \\
\underline{\underline{\underline{T}}} \\
\underline{\underline{1}}\end{array}$ & $\begin{array}{l}\frac{0}{0} \\
\frac{0}{0} \\
\underline{\underline{\underline{T}}} \\
\underline{\underline{1}}\end{array}$ & $\begin{array}{l}\frac{0}{0} \\
\frac{1}{0} \\
\frac{\underline{D}}{ \pm} \\
\underline{\underline{5}}\end{array}$ & $\begin{array}{l}\frac{0}{0} \\
\frac{1}{0} \\
\frac{\underline{\underline{D}}}{\leq} \\
\underline{\underline{5}}\end{array}$ & 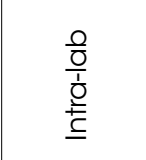 & $\begin{array}{l}\frac{0}{0} \\
\frac{0}{\dot{b}} \\
\stackrel{0}{0} \\
\stackrel{0}{U}\end{array}$ & $\begin{array}{l}\frac{0}{\frac{0}{0}} \\
\frac{1}{\frac{0}{\underline{D}}} \\
\underline{\underline{5}}\end{array}$ \\
\hline 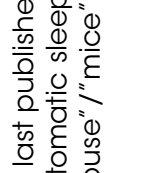 & 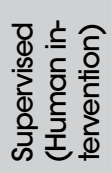 & $\stackrel{\mathscr{P}}{>}$ & $\stackrel{\circ}{z}$ & $\stackrel{\mathscr{山}}{\boldsymbol{\nu}}$ & $\stackrel{\stackrel{\sim}{>}}{>}$ & $\stackrel{\mathscr{P}}{>}$ & $\stackrel{\circ}{z}$ & $\stackrel{\mathscr{m}}{>}$ & 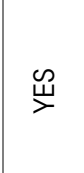 & $\stackrel{\circ}{z}$ & $\stackrel{\circ}{z}$ & $\stackrel{\mathscr{山}}{>}$ \\
\hline 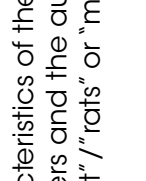 & 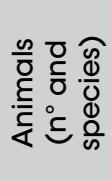 & 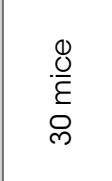 & $\frac{\omega}{\frac{0}{0}}$ & $\frac{\infty}{\frac{\infty}{0}}$ & $\frac{0}{0}$ & $\frac{0}{0}$ & $\frac{\infty}{0}$ & 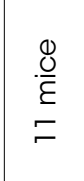 & 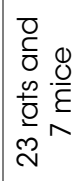 & $\begin{array}{l}\frac{0}{0} \\
\frac{.0}{\varepsilon} \\
a\end{array}$ & 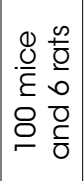 & $\begin{array}{l}\stackrel{0}{ } \\
\frac{U}{E} \\
\dot{I}\end{array}$ \\
\hline 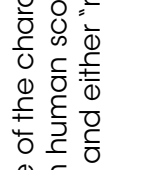 & 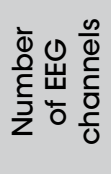 & $m$ & $\sim$ & - & $m$ & $\sim$ & $N$ & $\sim$ & $\nabla$ & - & - & $\sim$ \\
\hline 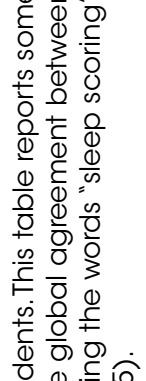 & $\begin{array}{l}\frac{0}{0} \\
0 \\
\frac{1}{10} \\
\sum\end{array}$ & 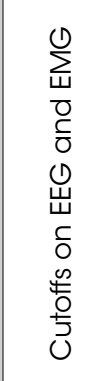 & 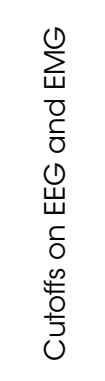 & 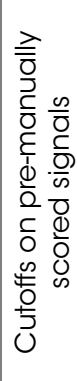 & 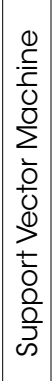 & 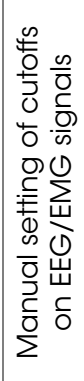 & 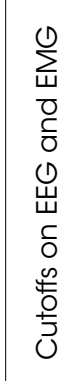 & 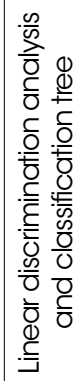 & 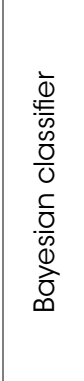 & 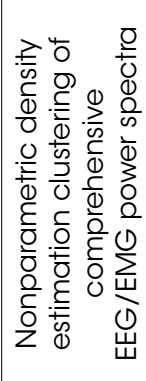 & 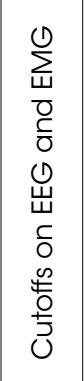 & 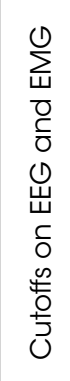 \\
\hline 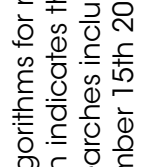 & 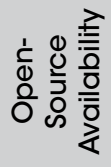 & $\stackrel{\mathscr{\nu}}{\underset{\nu}{ }}$ & O & O & $\frac{0}{z}$ & $\stackrel{\mathscr{\nu}}{\boldsymbol{\nu}}$ & 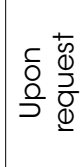 & 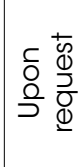 & $\stackrel{\mathscr{\nu}}{>}$ & $\stackrel{\mathscr{W}}{\boldsymbol{\nu}}$ & $\stackrel{\mathscr{W}}{\boldsymbol{\nu}}$ & 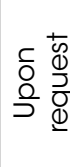 \\
\hline 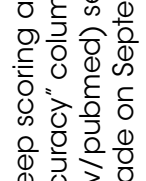 & 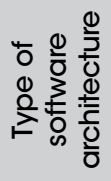 & 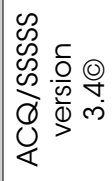 & 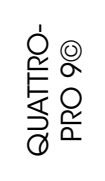 & $\begin{array}{l}\stackrel{3}{\mathrm{~m}} \\
\frac{0}{0} \\
\frac{1}{3}\end{array}$ & 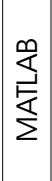 & 亭 & 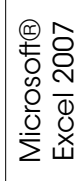 & 站 & 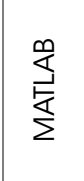 & 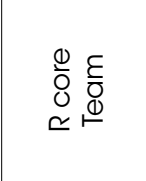 & $\frac{m}{\substack{S \\
\Sigma}}$ & 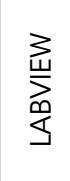 \\
\hline 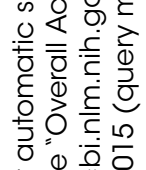 & 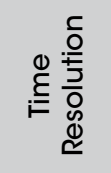 & $\stackrel{\infty}{\infty}$ & దొ & 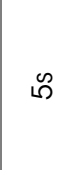 & 尺̊ & 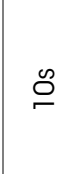 & దొ & $\frac{\infty}{\dot{\gamma}}$ & $\stackrel{\mathscr{\rho}}{q}$ & $\infty$ & कo & $\begin{array}{l}\frac{1}{0} \\
0 \\
0 \\
0 \\
0 \\
\frac{0}{2}\end{array}$ \\
\hline $\begin{array}{ll}0 & \\
0 & 0 \\
0 & 0 \\
3 & 0\end{array}$ & $\begin{array}{l}\bar{o} \\
\stackrel{D}{>}\end{array}$ & ষ্ণ & రి & ষ্ণ & 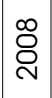 & 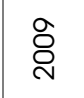 & ठ্े & $\stackrel{\circ}{\circ}$ & $\overline{\bar{n}}$ & $\bar{n}^{m}$ & $\frac{\nabla}{\sigma}$ & $\stackrel{\infty}{\stackrel{\sim}{2}}$ \\
\hline 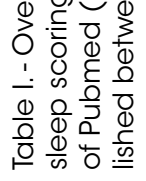 & 嵩 & 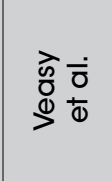 & 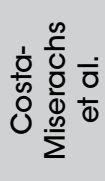 & 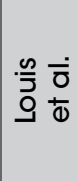 & 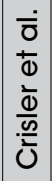 & $\begin{array}{l}\dot{\overline{0}} \\
\overline{0} \\
0 \\
00 \\
0 \\
\mathbb{0}\end{array}$ & 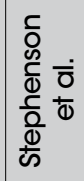 & 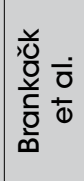 & 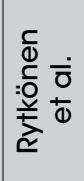 & 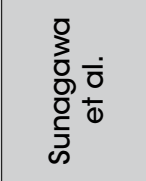 & 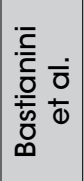 & 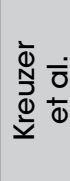 \\
\hline
\end{tabular}


Indeed, sleep pathophysiology touches upon derangements at the molecular level (Dauvilliers and Tafti, 2008) as well as to the level of more complex physiological systems (Silvani et al., 2013; Spiegel et al., 2009). Consequently, it is likely that many researchers whose primary interests are distant from the sleep field will at some point include sleep in their experimental projects. Since a significant part of sleep research is performed on rodents, the development of an automatic algorithm to discriminate wake-sleep states validated across different laboratories and species would be of great help. The advantages in using an automatic algorithm for sleep scoring in rodents are not limited to the acceleration of the studies, but also include increased objectiveness and reproducibility of the results. Many automatic algorithms for sleep scoring have been proposed in the last few years (Brankack et al., 2010; Crisler et al., 2008; Gross et al., 2009; Kohn et al., 1974; Louis et al., 2004; Rytkonen et al., 2011; Sunagawa et al., 2013; Veasey et al., 2000). However, even those with excellent performances have not been validated across different laboratories or animal species (Table I). Recently, we have published SCOPRISM (Bastianini et al., 2014), a new automatic algorithm for sleep scoring validated both in mice and rats on datasets of 3 different laboratories and we made its script freely available. We thus believe that SCOPRISM could represent a first step in the development of a widely diffused standard automatic algorithm for sleep scoring.

\section{Acknowledgements}

This project was supported by the University of Bologna (FARB line 1 grant code FFBO120705, to GZ; FARB line 2 grant code RFBO124043, to AS).

\section{References}

Amici R., Bastianini S., Berteotti C., Cerri M., Del Vecchio F., Lo Martire V., Luppi M., Perez E., Silvani A., Zamboni G., Zoccoli G. Sleep and bodily functions: the physiological interplay between body homeostasis and sleep homeostasis. Arch. Ital. Biol., 152: 66-78, 2014.

Bastianini S., Berteotti C., Gabrielli A., Del Vecchio F., Amici R., Alexandre C., Scammell T.E., Gazea M., Kimura M., Lo Martire V., Silvani A., Zoccoli
G. SCOPRISM: a new algorithm for automatic sleep scoring in mice. J. Neurosci. Methods, 235: 277-284, 2014.

Bastianini S., Silvani A., Berteotti C., Elghozi J.L., Franzini C., Lenzi P., Lo Martire V., Zoccoli G. Sleep related changes in blood pressure in hypocretin-deficient narcoleptic mice. Sleep, 34: 213-218, 2011.

Bastianini S., Silvani A., Berteotti C., Lo Martire V., Zoccoli G. High-amplitude theta wave bursts during REM sleep and cataplexy in hypocretindeficient narcoleptic mice. J. Sleep Res., 21: 185188, 2012.

Bergmann B.M., Mistlberger R.E., Rechtschaffen A. Period-amplitude analysis of rat electroencephalogram: stage and diurnal variations and effects of suprachiasmatic nuclei lesions. Sleep, 10: 523-536, 1987.

Brankack J., Kukushka V.I., Vyssotski A.L., Draguhn A. EEG gamma frequency and sleep-wake scoring in mice: comparing two types of supervised classifiers. Brain Res., 1322: 59-71, 2010.

Cerri M., Mastrotto M., Tupone D., Martelli D., Luppi M., Perez E., Zamboni G., Amici R. The inhibition of neurons in the central nervous pathways for thermoregulatory cold defense induces a suspended animation state in the rat. J. Neurosci., 33: 2984-2993, 2013.

Chemelli R.M., Willie J.T., Sinton C.M., Elmquist J.K., Scammell T., Lee C., Richardson J.A., Williams S.C., Xiong Y., Kisanuki Y., Fitch T.E., Nakazato M., Hammer R.E., Saper C.B., Yanagisawa M. Narcolepsy in orexin knockout mice: molecular genetics of sleep regulation. Cell, 98: 437-451, 1999.

Crisler S., Morrissey M.J., Anch A.M., Barnett D.W. Sleep-stage scoring in the rat using a support vector machine. J. Neurosci. Methods, 168: 524-534, 2008.

Dauvilliers Y., Tafti M. The genetic basis of sleep disorders. Curr. Pharm. Des., 14: 3386-3395, 2008.

Dengler V., Westphalen K., Koeppen M. Disruption of circadian rhythms and sleep in critical illness and its impact on innate immunity. Curr. Pharm. Des., 21: 3349-3476, 2015.

Diniz Behn C.G., Klerman E.B., Mochizuki T., Lin S.C., Scammell T.E. Abnormal sleep/wake dynamics in orexin knockout mice. Sleep, 33: 297306, 2010.

El Helou J., Belanger-Nelson E., Freyburger M., Dorsaz S., Curie T., La Spada F., Gaudreault P.O., Beaumont E., Pouliot P., Lesage F., Frank M.G., 
Franken P., Mongrain V. Neuroligin-1 links neuronal activity to sleep-wake regulation. Proc. Natl. Acad. Sci.U.S.A., 110: 9974-9979, 2013.

Floras J.S. Hypertension and Sleep Apnea. Can. J. Cardiol., 31: 889-897, 2015.

Glin L., Arnaud C., Berracochea D., Galey D., Jaffard R., Gottesmann C. The intermediate stage of sleep in mice. Physiol. Behav., 50: 951-953, 1991.

Gondard E., Anaclet C., Akaoka H., Guo R.X., Zhang M., Buda C., Franco P., Kotani H., Lin J.S. Enhanced histaminergic neurotransmission and sleep-wake alterations, a study in histamine H3-receptor knock-out mice. Neuropsychopharmacology, 38: 1015-1031, 2013.

Gottesmann C. The transition from slow-wave sleep to paradoxical sleep: evolving facts and concepts of the neurophysiological processes underlying the intermediate stage of sleep. Neurosci. Biobehav. Rev., 20: 367-387, 1996.

Grimaldi D., Calandra-Buonaura G., Provini F., Agati P., Pierangeli G., Franceschini C., Barletta G., Plazzi G., Montagna P., Cortelli P. Abnormal sleep-cardiovascular system interaction in narcolepsy with cataplexy: effects of hypocretin deficiency in humans. Sleep, 35: 519-528, 2012.

Gross B.A., Walsh C.M., Turakhia A.A., Booth V., Mashour G.A., Poe G.R. Open-source logicbased automated sleep scoring software using electrophysiological recordings in rats. J. Neurosci. Methods, 184: 10-18, 2009.

Hara J., Beuckmann C.T., Nambu T., Willie J.T., Chemelli R.M., Sinton C.M., Sugiyama F., Yagami K., Goto K., Yanagisawa M., Sakurai T. Genetic ablation of orexin neurons in mice results in narcolepsy, hypophagia, and obesity. Neuron, 30: 345-354, 2001.

Kantor S., Mochizuki T., Lops S.N., Ko B., Clain E. Clark E., Yamamoto M., Scammell T.E. Orexin gene therapy restores the timing and maintenance of wakefulness in narcoleptic mice. Sleep, 36: 1129-1138, 2013.

Kohn M., Litchfield D., Branchey M., Brebbia D.R. An automatic hybrid analyzer of sleep stages in the rat. Electroencephalogr. Clin. Neurophysiol. 37: 518-520, 1974.

Kreuzer M., Polta S., Gapp J., Schuler C., Kochs E.F., and Fenzl T. Sleep scoring made easy-Semiautomated sleep analysis software and manual rescoring tools for basic sleep research in mice. MethodsX, 2: 232-240, 2015.

Libourel P.A., Corneyllie A., Luppi P.H., Chouvet G., Gervasoni D. Unsupervised online classifier in sleep scoring for sleep deprivation studies. Sleep, 38: 815-828, 2015.

Louis R.P., Lee J., Stephenson R. Design and validation of a computer-based sleep-scoring algorithm. J. Neurosci. Methods, 133: 71-80, 2004.

Luppi P.H., Peyron C., Fort P. Role of MCH neurons in paradoxical (REM) sleep control. Sleep, 36: 1775-1776, 2013.

Mouse Genome Sequencing C., Waterston R.H., Lindblad-Toh K., Birney E., Rogers J., Abril J.F., Agarwal P., Agarwala R., Ainscough R., Alexandersson M., An P., Antonarakis S.E., Attwood J., Baertsch R., Bailey J., Barlow K., Beck S., Berry E., Birren B., Bloom T., Bork P., Botcherby M., Bray N., Brent M.R., Brown D.G., Brown S.D., Bult C., Burton J., Butler J., Campbell R.D., Carninci P., Cawley S., Chiaromonte F., Chinwalla A.T., Church D.M., Clamp M., Clee C., Collins F.S., Cook L.L., Copley R.R., Coulson A., Couronne O., Cuff J., Curwen V., Cutts T., Daly M., David R., Davies J., Delehaunty K.D., Deri J., Dermitzakis E.T., Dewey C., Dickens N.J., Diekhans M., Dodge S., Dubchak I., Dunn D.M., Eddy S.R., Elnitski L., Emes R.D., Eswara P., Eyras E., Felsenfeld A., Fewell G.A., Flicek P., Foley K., Frankel W.N., Fulton L.A., Fulton R.S., Furey T.S., Gage D., Gibbs R.A., Glusman G., Gnerre S., Goldman N., Goodstadt L., Grafham D., Graves T.A., Green E.D., Gregory S., Guigo R., Guyer M., Hardison R.C., Haussler D., Hayashizaki Y., Hillier L.W., Hinrichs A., Hlavina W., Holzer T., Hsu F., Hua A., Hubbard T., Hunt A., Jackson I., Jaffe D.B., Johnson L.S., Jones M., Jones T.A., Joy A., Kamal M., Karlsson E.K., Karolchik D., Kasprzyk A., Kawai J., Keibler E., Kells C., Kent W.J., Kirby A., Kolbe D.L., Korf I., Kucherlapati R.S., Kulbokas E.J., Kulp D., Landers T., Leger J.P., Leonard S., Letunic I., Levine R., Li J., Li M., Lloyd C., Lucas S., Ma B., Maglott D.R., Mardis E.R., Matthews L., Mauceli E., Mayer J.H., McCarthy M., McCombie W.R., McLaren S., McLay K., McPherson J.D., Meldrim J., Meredith B., Mesirov J.P., Miller W., Miner T.L., Mongin E., Montgomery K.T., Morgan M., Mott R., Mullikin J.C., Muzny D.M., Nash W.E., Nelson J.O., Nhan M.N., Nicol R., Ning Z., Nusbaum C., O'Connor M.J., Okazaki Y., Oliver K., Overton-Larty E., Pachter L., Parra G., Pepin K.H., Peterson J., Pevzner P., Plumb R., Pohl C.S., Poliakov A., Ponce T.C., Ponting C.P., Potter S., Quail M., Reymond A., Roe B.A., Roskin K.M., Rubin E.M., Rust A.G., Santos R., Sapojnikov V., Schultz B., Schultz J., Schwartz M.S., Schwartz S., Scott C., Seaman S., Searle S., Sharpe T., Sheridan A., Shownkeen R., Sims S., 
Singer J.B., Slater G., Smit A., Smith D.R., Spencer B., Stabenau A., Stange-Thomann N., Sugnet C., Suyama M., Tesler G., Thompson J., Torrents D., Trevaskis E., Tromp J., Ucla C., Ureta-Vidal A., Vinson J.P., Von Niederhausern A.C., Wade C.M., Wall M., Weber R.J., Weiss R.B., Wendl M.C., West A.P., Wetterstrand K., Wheeler R., Whelan S., Wierzbowski J., Willey D., Williams S., Wilson R.K., Winter E., Worley K.C., Wyman D., Yang S., Yang S.P., Zdobnov E.M., Zody M.C., Lander E.S. Initial sequencing and comparative analysis of the mouse genome. Nature, 420: 520-562, 2002.

Palagini L., Drake C.L., Gehrman P., Meerlo P., Riemann D. Early-life origin of adult insomnia: does prenatal-early-life stress play a role? Sleep Med., 16: 446-456, 2015.

Paterson L.M., Nutt D.J., Wilson S.J. Sleep and its disorders in translational medicine. $J$. Psychopharmacol., 25: 1226-1234, 2011.

Robert C., Guilpin C., Limoge A. Automated sleep staging systems in rats. J. Neurosci. Methods, 88: 111-122, 1999.

Romeijn N., Raymann R.J., Most E., Te Lindert B., Van Der Meijden W.P., Fronczek R., Gomez-Herrero G., Van Someren E.J. Sleep, vigilance, and thermosensitivity. Pflugers Arch., 463: 169-176, 2012.

Ronzhina M., Janousek O., Kolarova J., Novakova M., Honzik P., Provaznik I. Sleep scoring using artificial neural networks. Sleep Med. Rev., 16: 251-263, 2012.

Rytkonen K.M., Zitting J., Porkka-Heiskanen T. Automated sleep scoring in rats and mice using the naive Bayes classifier. J. Neurosci. Methods, 202: 60-64, 2011.

Sateia M.J. International classification of sleep disorders-third edition: highlights and modifications. Chest, 146: 1387-1394, 2014.
Sharma R., Sahota P., Thakkar M.M. Role of adenosine and the orexinergic perifornical hypothalamus in sleep-promoting effects of ethanol. Sleep, 37: 525-533, 2014.

Silvani A., Bastianini S., Berteotti C., Cenacchi G., Leone O., Lo Martire V., Papa V., Zoccoli G. Sleep and cardiovascular phenotype in middle-aged hypocretin-deficient narcoleptic mice. $J$. Sleep Res. 23: 98-106, 2014.

Silvani A., Grimaldi D., Barletta G., Bastianini S., Vandi S., Pierangeli G., Plazzi G., Cortelli P. Cardiovascular variability as a function of sleepwake behaviour in narcolepsy with cataplexy. $J$. Sleep Res., 22: 178-184, 2013.

Spiegel K., Tasali E., Leproult R., Van Cauter E. Effects of poor and short sleep on glucose metabolism and obesity risk. Nat. Rev. Endocrinol., 5: 253-261, 2009.

Sunagawa G.A., Sei H., Shimba S., Urade Y., Ueda H.R. FASTER: an unsupervised fully automated sleep staging method for mice. Genes to Cells, 18 : 502-518, 2013.

Toth L.A., Bhargava P. Animal models of sleep disorders. Comp. Med., 63: 91-104, 2013.

Vassalli A., Dellepiane J.M., Emmenegger Y., Jimenez S., Vandi S., Plazzi G., Franken P., Tafti M. Electroencephalogram paroxysmal theta characterizes cataplexy in mice and children. Brain, 136: 1592-1608, 2013.

Veasey S.C., Valladares O., Fenik P., Kapfhamer D., Sanford L., Benington J., Bucan M. An automated system for recording and analysis of sleep in mice. Sleep, 23: 1025-1040, 2000.

Vivaldi E.A., Bassi A. Frequency domain analysis of sleep EEG for visualization and automated state detection. Conf. Proc. IEEE Eng. Med. Biol. Soc., 1: 3740-3743, 2006. 